\title{
Extension of Reflection-Mode Digital Gradient Sensing Method for Visualizing and Quantifying Transient Deformations and Damage in Solids
}

\author{
Amith S. Jain and Hareesh V. Tippur ${ }^{1}$ \\ Department of Mechanical Engineering \\ Auburn University, AL 36849, USA
}

\begin{abstract}
The reflection-mode Digital Gradient Sensing (r-DGS) method is extended to visualize and quantify dynamic deformations in solids due to stress wave propagation and for damage/disbond detection in layered materials due to impact loading. The r-DGS technique employs digital image correlation principles to quantify two orthogonal surface slopes simultaneously in specularly reflective solids. Here, for the first time, r-DGS has been implemented in conjunction with ultrahigh-speed photography to quantitatively map surface slopes during stress wave propagation in a thin plate due to an impact event. The measured surface slopes have also been used to successfully evaluate instantaneous topographic information. The r-DGS method has been subsequently demonstrated to be an effective tool for detecting disbonds in layered structures subjected to impact loading. Baseline experiments in each category have been carried out to demonstrate the methodology.
\end{abstract}

Keywords: optical metrology, surface slopes, digital image correlation, stress wave propagation, high-speed photography, damage detection

\section{Introduction}

Thin structures experience rapid loading in a variety of engineering scenarios. Examples include tool drop and pebble impact on airframe panels, hail damage of windows and automotive windshields, etc. Visualization and quantification of deformations and damage in these and other similar situations is important for failure characterization and non-destructive evaluation (NDE) of solids and structures. In thin structures, elastic stresses are proportional to curvatures and hence quantifying them accurately would help assess their mechanical response and integrity when used in conjunction with failure theories. Measuring surface slopes directly using non-

\footnotetext{
${ }^{1}$ McWane Professor and corresponding author, (Tel: +1-334-844-3327, email: tippuhv@auburn.edu) 
contact, full-field methods is valuable for such applications. That is, slope measurements offer unique advantages over measuring out-of-plane displacements because a single step integration or differentiation of measured data could yield topography or curvatures, respectively, of thin structures. In doing so, numerical errors due to two successive differentiations of displacements can be avoided if curvatures were to be determined using measured out-of-plane displacements.

Several techniques have been proposed for measuring surface slopes and curvatures of solids over the years. They can be broadly classified into incoherent and coherent methods based on white light or laser light illumination. Among the former type, the slope measurements based on Lichtenberg's reflection moiré concept [1] has taken hold in numerous forms. Kao and Chiang [2] have described several such moiré methods for visualizing and quantifying surface slopes and curvatures. Ritter [3] has demonstrated the method for studying dynamic problems including vibrating plates. Optical differentiation by superposing spatially shifted moiré fringes representing displacements and slopes were also discussed. Among the grating-based real-time interferometers, the early work of Assa et al [4] is notable. They have proposed a shearing interferometer for measuring slopes and curvatures which can account for the initial curvature of the model. The works of Tippur and his co-workers resulted in a grating-based lateral shearing interferometry called the Coherent Gradient Sensing (CGS) [5] method to quantify real-time surface slopes and curvatures [6-8] of thin films and structures. Many other double exposure and single exposure techniques [9-14] are also reported in the literature for measuring slopes and curvatures of specularly and diffusely reflective surfaces.

Recently, digital image correlation methods have become very popular for measuring 2D and 3D deformations [15-19] in a variety of static and dynamic experimental mechanics problems. In this context, Periasamy and Tippur have recently proposed a new method called Digital Gradient Sensing (DGS) [20-21] based on digital image correlation principles for measuring angular deflections of light rays in planar phase objects. They have related angular deflections of light rays to planar stress gradients. Subsequently, they have also modified DGS to study reflective objects by mapping orthogonal surface slopes and curvatures simultaneously [22]. The simplicity of its optical arrangement, ubiquitous commercial and freeware image correlation software packages, and technological advances in terms of high pixel and gray scale resolutions of sensors can all be readily leveraged to further advance this new approach for 
experimental mechanics and NDE. Further, the method can be applied in principle using other wavelengths of light such as infrared circumventing the limitation of object surface specularity in visible wave lengths. In view of this, the current work deals with extending DGS to slope measurement in thin structures during stress-wave dominant situations and for impact induced damage detection.

\section{Optical Setup and Working Principle}

The schematic of the optical setup used for reflection-mode DGS (or, r-DGS) is shown in Fig. 1. It consists of a specularly reflective planar object (specimen), a 50/50 beam splitter, a randomly speckled/textured planar target and a digital recording device (camera). The speckle pattern on the target is prepared by decorating a planar surface with alternative black/white mists of paint. The nature of speckles used for a given application depends on the camera parameters (pixel and gray scale resolutions, camera gain, numerical aperture of the lens), magnification and measurement resolution needed.

The optical arrangement is such that the digital camera is used to record speckles on the target plane via the reflective specimen and the beam splitter. In the undeformed or the reference state of the specimen, a generic point $\mathrm{P}$ on the target is photographed via a point $\mathrm{O}$ on the specimen surface. When the specimen deforms, the point $\mathrm{O}$ moves to $\mathrm{O}$ ' with an out-of-plane displacement $w$ in the $z$-direction (optical axis). This results in the camera photographing a neighboring point $\mathrm{Q}$ on the target in the deformed state of the specimen. By correlating the two images corresponding to the reference and deformed states of the specimen, the local displacement components $\delta_{x}$ and $\delta_{y}$ in the $x$ - and $y$-directions, respectively, can be evaluated in the whole field of observation. Knowing the local surface normal at Q, the surface slope components in the $x-z$ and $y-z$ planes can be evaluated as [22],

$$
\phi_{x ; y} \approx \tan \phi_{x ; y}=2 \frac{\partial w}{\partial x ; y}=\frac{\delta_{x ; y}}{\Delta}
$$

where $\phi_{x ; y}$ represent angular deflections of light rays. Here $\Delta$ denotes the distance between the specimen and target planes and is the optical lever that controls the sensitivity of the method in 
addition to those associated with 2D digital image correlation such as the sub-image size, pixel overlap and the searching algorithm used.

\section{Baseline Experiments}

In order to calibrate the experimental methodology, first an experiment on a thin plate subjected to a central deflection was carried out. This experiment was also used to validate experimental repeatability and test different experimental parameters such as the type of speckles and the target distance used during the experiments. A $280 \mu \mathrm{m}$ thick, $50 \mathrm{~mm}$ diameter silicon wafer was bonded to a thick steel washer with a clear aperture of $25 \mathrm{~mm}$ diameter co-axially using an epoxy adhesive. The silicon wafer was subjected to a central deflection using a point load applied using a micrometer stem ( $\sim 3 \mathrm{~mm}$ diameter with a hemispherical tip). Different magnitudes of deflections, 10, 20 and $30 \mu \mathrm{m}$ were applied during the experiment. The speckle images corresponding to the reference as well as the deformed states were recorded using a Nikon D100 camera with a CCD sensor set to 1504 x 1000 pixels and 8 bit gray scale resolution. A long focal length lens $(70-300 \mathrm{~mm})$ attached to an adjustable bellows was used to focus on the target plane speckles via the reflective silicon wafer surface. The experimental parameters $L$ and $\Delta$ in these experiments were set at $1217 \mathrm{~mm}$ and $65 \mathrm{~mm}$, respectively. Here $L$ denotes the distance between the specimen surface and the target plane. An example of a pair of such recordings is shown in Fig. 2 for an applied central deflection of $30 \mu \mathrm{m}$.

The speckle images were correlated (using 2D digital image correlation software ARAMIS) by segmenting them into 20 x 20 pixels sub-images ( 1 pixel covered $39.6 \mu \mathrm{m}^{2}$ on the target plane). The resulting displacements $\delta_{x}$ and $\delta_{y}$ were used to calculate the angular deflections of light rays $\phi_{x ; y}$ for each sub-image and hence the corresponding surface slopes $\frac{\partial w}{\partial x ; y}$. Spatial contours of the two orthogonal surface slopes plotted in increments of $5 \times 10^{-4}$ rad are shown in Fig. 3 as solid lines. (It should be noted that the coordinates of the target plane

were mapped to that of the silicon wafer using $(x ; y)=\frac{L}{L+\Delta}\left(x_{0} ; y_{0}\right)$ where $L$ is the distance between the camera and the specimen and subscript ' 0 ' denotes coordinates of the target plane 
[22].) Analytically evaluated orthogonal slopes for a thin circular plate subjected to a central deflection given by,

$$
\frac{\partial w}{\partial x ; y}=\frac{4(x ; y) w}{R^{2}} \log \left(\frac{\sqrt{x^{2}+y^{2}}}{R}\right)
$$

are also plotted in Fig. 3 as broken lines for a direct comparison with the experimental data. A very good agreement between the two sets of results is evident in the region of interest.

An important part of the r-DGS methodology is the target plane speckles used during experimentation. To further examine this, the experiments described above were repeated using three different speckle targets, qualitatively categorized as fine, medium, and coarse. Fig. 4 shows recordings of all the three speckle patterns used in this experiment. In these speckles covered 4-5 pixels, 11-12 pixels and 22-24 pixels on average for the fine, medium and coarse cases, respectively. The slope contours $\frac{\partial w}{\partial y}$ for an applied displacement of $20 \mu \mathrm{m}$ are shown Fig. 5(a). These results were obtained by analyzing speckle image pairs in the reference and deformed states by segmenting them into 20 x 20 pixel sub-images (1 pixel covered $41.0 \mu^{2}$ for fine speckles, $38.8 \mu \mathrm{m}^{2}$ for medium speckles, and $39.6 \mu \mathrm{m}^{2}$ for coarse speckles on the target plate $^{2}$ ) during image correlation performed without any pixel overlap. From the figure it can be seen that despite the differences in the nature of speckles, very good repeatability exists ${ }^{3}$ in the measured values of surface slopes from all the three speckle targets. A further confirmation of the same is evident in the quantitative plot of surface slopes along the vertical diameter of the wafer for all three speckle targets used.

The angular deflections in r-DGS relies on spatial domain correlation of reference and deformed speckle images, hence the sub-image size plays a role in the evaluation of surface slope components. The sub-image size chosen was based on achieving correlation at all points as

\footnotetext{
${ }^{2}$ Ideally the same magnification in all the three cases is preferable but swapping out the targets plates after each experiment required refocussing. This in turn marginally altered the magnification factor during experiments.

${ }^{3}$ Expectedly, the green contour representing the zero slope level is the one most sensitive to errors including speckle noise. Since DGS employs DIC, the existing literature on the role of speckle noise on measurement errors are relevant here as well.
} 
it was necessary to have slope components available in the entire field in order to be able to perform numerical integration to obtain surface topography (to be discussed next). The larger sub-image size produces higher averaging effect and smaller data array in the field of view without pixel overlap compared to the smaller sub-image size [19]. Hence the trade-offs, if any, were studied. Fig. 6 shows the measured $\frac{\partial w}{\partial y}$ contours for the medium size speckle target used for three different sub-image sizes of $15 \times 15,20 \times 20$, and $25 \times 25$ pixels. Again, a good agreement between the slope contours can be seen in the figure. Marginal increase in noise is evident for the contours of zero slope whereas the higher ones are largely unaffected within experimental errors. As expected the images with smaller sub-image sizes have higher noise compared to the larger sub-images due to averaging effects and produce smoother contours. Again the zero slope contours are affected the most (the deviation between the upper and lower halves could be possibly attributed to the experimental arrangement and/or uniformity of illumination through the beam splitter).

Experiments for different target distances have also been performed while keeping all other parameters $(L=1217 \mathrm{~mm})$ the same. Again, a very good reproducibility of surface slopes was observed. A pair of these results for $\Delta$ values of $65 \mathrm{~mm}$ and $110 \mathrm{~mm}$ is shown in Fig. 5(c). Again, good agreement in the two measurements is clearly evident for the $\frac{\partial w}{\partial y}$ contours shown. Further details are avoided here for brevity but can be found in Ref. 23.

In reflection-mode DGS, $\delta_{x}$ and $\delta_{y}$ measurements being proportional to surface slopes, $\frac{\partial w}{\partial x}$ and $\frac{\partial w}{\partial y}$ can be numerically integrated with respect to the in-plane spatial coordinates to estimate topographic information of the deformed surface. In this work, the trapezoidal rule of integration was used to obtain out-of-plane deformations. The regions outside the clamped zone (adhesively bonded circumference) were assumed to have a zero out-of-plane displacement. Integration was carried along both the in-plane ( $x$ and $y$ ) directions independently. The resulting surface topographs are shown in Fig. 7. The results from integration show a rather close agreement (within 5\%) with the maximum value imposed at the center of the plate in these experiments. The integrated values have a peak value marginally lower than the applied 
displacement in each case. This is attributed to the micrometer backlash and/or propagation of errors in the simple integration scheme employed.

\section{Mapping Impact Induced Deformations}

Periasamy and Tippur [22] have shown that r-DGS used in conjunction with time-lapse photography could be used to monitor evolution of surface slopes during slow speed events such as warping of a silicon wafer during in-situ room temperature curing of a thin polymer coating. In this work, the feasibility of the method to study deformation of thin plate-like structures when subjected to stress-wave dominant loading conditions is demonstrated using high-speed photography.

A PMMA (poly-methyl methacrylate) disk of $3 \mathrm{~mm}$ thickness and $76.2 \mathrm{~mm}$ diameter was used for conducting an impact loading experiment. One face of the disk was made specularly reflective by sputter coating it with aluminum using vacuum deposition technique. The schematic of the experimental setup for r-DGS is as shown in Fig. 8. The target plate was placed at a distance $(\Delta)$ of $110 \mathrm{~mm}$ from the disk. The PMMA disk facing its reflective side towards the camera was held coaxially on one side of a thick rectangular steel plate having a circular aperture using three sticky 3M soft-gel dots ( $\sim 3 \mathrm{~mm}$ diameter) to mimic 'free-free' boundary conditions. A steel ball of diameter $25.4 \mathrm{~mm}$ travelling at a velocity of $2.3 \mathrm{~m} / \mathrm{sec}$ was used to impact the center of the PMMA disk. The impactor was a spherical ball pendulum held at rest by an electromagnet operated by an open/close DC circuit. A Cordin-550 multi-sensor high-speed digital camera was used for recording speckle images at a rate of 80,425 frames per second during stress wave propagation event. The high-speed camera was equipped with 32, $1000 \mathrm{x}$ 1000 pixel, 8 bit CCD sensors and was capable of capturing images sequentially upon receiving an initial trigger produced by a photo-gate device used in conjunction with the pendulum impactor. A pair of high-intensity flash lamps triggered by the camera produced the required illumination for recording the speckle images. Two sets of 32 images, one captured before the impact event and the other during the impact event, were recorded. The images recorded by the same sensor in each set of images were paired and correlated using digital image correlation software ARAMIS. Coarse speckles were used in these experiments due to a relatively small numerical aperture (\#32) and a larger (compared to the quasi-static experiments) target distance. This also allowed the camera gain settings to be in the middle of the 8 bit range of the sensor 
avoiding pixel saturation and achieve optimum exposure. Examples of select speckle images for six different time instants recorded during the impact event are shown in Fig. 9. Evidence of the impact event and the resulting speckle motion can be seen in the central part of images at later time instants following initial impact at $t=0$.

The images in the reference and deformed sets were segmented into $30 \times 30$ pixel subimages (1 pixel covered $76 \mu \mathrm{m}^{2}$ on the target plate) while correlating images and no overlap was used during analysis. Here a larger sub-image size was chosen in order to achieve correlation over the entire image as coarse speckles were employed in these experiments. The resulting time-resolved contour plots of angular deflections of light rays in the $x-z$ and $y-z$ planes and the corresponding surface slopes due to transient stress wave propagation in the circular plate are shown in Figs. 10(a) and (b), respectively. In these, the frame immediately prior to the one where surface slope contours are evident is associated with the start of the impact event or $t=0$. There is a qualitative resemblance between these contours with the quasi-static counterparts shown in Fig. 3. The longitudinal wave speed in PMMA is approximately $2700 \mathrm{~m} / \mathrm{sec}$ [24]. Thus, the impact induced stress waves emanating from the impact point take $\sim 30 \mu$ sec to reach the outer edge of the circular disk and another $\sim 30 \mu$ sec thereafter for the reflected waves from the edges to return to the point of impact, identified as the origin in these images. In view of this, deformations in the early stages of impact are highly localized, close to the origin, with the rest of the plate undeformed and hence show a zero slope value. This situation is akin to a thin circular plate clamped/bonded along its periphery and subjected to a central deflection, as in the quasi-static silicon wafer experiment discussed earlier. Accordingly, the two orthogonal surface slope contours shown in Figs. 10(a) and (b) resemble those in Fig. 3. Further, the vectorial sum of the two measurements, shown in Fig. 11, depicts the expected axisymmetric nature of deformations relative to the point of impact. Increasing number of contours between two successive images indicate the highly transient deformations as the stress waves propagate in the disk. Further, the positive resultant slopes, in Fig. 11 show a nearly constant (maximum) slope bounded by regions of zero slope along the outer edges of the deformed region and the point of impact.

The surface slopes were numerically integrated as in the quasi-static counterpart to estimate the instantaneous out-of-plane displacements and the plate topography. Thus obtained 
out-of-plane displacements $(w)$ along the horizontal and vertical radii of the disk, evaluated from the data for each sub-image, are plotted in Fig. 12 as solid symbols.

A companion 3D elasto-dynamic finite element simulation was performed using ANSYS $^{\mathrm{TM}}$ Workbench software package and a quarter-model was simulated. The model (Fig. 13) was discretized into 500095 quadratic explicit elements with 518718 nodes. Frictionless interaction was defined between the two solid bodies. The dynamic elastic modulus and Poisson's ratio were assigned as 5.8 GPa and 0.27 respectively to the PMMA disk based on ultrasonic measurement of longitudinal and shear wave speeds and density. The velocity of the steel ball of $2.3 \mathrm{~m} / \mathrm{sec}$, measured by the photo-detector during the experiments, was used as an input in these simulations. The time steps during the analysis were allowed to be automatically controlled by the explicit integration scheme. Output was requested every $12 \mu$ sec to match the experimental time interval between two successive images of the high-speed camera. The computed data from the numerical simulation are superimposed on experimentally obtained outof-plane displacements in the Fig. 12. It can be readily seen that there is a good agreement between both data sets, demonstrating the feasibility of r-DGS for stress-wave dominant problems.

\section{Applications to Disbond and Damage Detection}

Next, the feasibility of r-DGS method to detect disbond and damage in layered plates was considered. First a disbond detection study was carried out on an adhesively bonded bi-layered PMMA plate. Subsequently its applicability was extended to detect damage in a glass fiber reinforced epoxy composite plate subjected to impact loading. For simplicity of implementation, thermal excitation method [25] was used in these experiments to induce thermo-mechanical deformations and enable detection of disbond and damage.

Two sets of experiments were performed to identify disbond and delamination in (a) thin plates bonded using an acrylic adhesive, and (b) on composite plates subjected to impact loading. A 2D schematic of the r-DGS experimental setup used is shown in Fig. 14. Specularly reflective test specimens were held vertically in a slotted screw-type sample holder (an optical mount consisting of two screws $40 \mathrm{~mm}$ apart) at the bottom edge. The bi-layered circular PMMA plates were held in the holder at a single point with the help of a screw whereas in the composite plate 
experiment, the sample was clamped at two points using the same holder. A beam splitter and a target plate coated with random speckles were arranged in front of the specimen to facilitate recording of speckles via the reflective face of the specimen. The target plate distance $(\Delta)$ was $105 \mathrm{~mm}$ for disbond detection and $65 \mathrm{~mm}$ for delamination detection experiments, respectively. A Nikon D100 digital SLR camera with 28-300 mm focal length lens was placed in front of the specimen to photograph the speckles. A $75 \mathrm{~W}$ incandescent lamp was placed behind the specimen at a distance of $\sim 100 \mathrm{~mm}$ to cause thermo-mechanical deformations. A reference image was recorded using a camera resolution of 1504 x 1000 pixels (one pixel covered $73 \mu^{2}$ and $43 \mu^{2}$ in disbond detection and delamination detection experiments, respectively) before turning on the incandescent lamp. Deformed image of the specimen was captured after 15 minutes of thermal excitation. The variation in the temperature on the back surface was $4^{\circ}-6^{\circ} \mathrm{C}$. The images corresponding to the deformed state along with the ones from the reference state was then used to extract the two orthogonal gradient fields using digital image correlation. As the specimen absorbed the thermal energy emitted by the incandescent lamp, the disbonded and damaged regions deformed anomalously relative to the rest of the specimen. That is, abrupt changes in surface slopes in an otherwise uniform r-DGS contour map occurred when slope fields were visualized.

\subsection{Disbond Detection}

Two isotropic PMMA plates of $\sim 1.5 \mathrm{~mm}$ thickness and $76.2 \mathrm{~mm}$ in diameter were used for this demonstration to prepare a bi-layered disk. The sample preparation involved two plates bonded together using an acrylic adhesive (Weldon 16). To create a disbond on the interface, one of the faces of PMMA plates was coated with a layer of mold release agent (from Buehler, Inc.) over approximately $25 \mathrm{~mm}$ diameter. The specimen was allowed to cure for a time period of 48 hours. One of the faces of the cured bi-layered disk was made specularly reflective by coating it with aluminum film ( 100 nm) using vacuum deposition. Two samples, nominally identical in geometry, one with a disbond and the other without any disbond were prepared for this comparative study. The disks were subjected to thermal loading using an incandescent lamp, as described earlier. The surface deformations due to thermal loading between the samples were examined to detect the disbond using abnormalities in the surface slope contours measured using r-DGS. 
Figures 15(a) and (b) show photographed speckle images representing the reference and thermally excited states of the specimen. Note that Fig. 15(b) corresponds to a gray scale recording following illuminating/heating the disks for 15 minutes. Figs. 15(c)-(f) show the resulting orthogonal surface slope contours from r-DGS due to thermo-mechanical deformation of the disks with and without disbond, respectively. During image correlation, the images were segmented into 15 x 15 pixel non-overlapping sub-images yielding an array of 99 x 65 data points.

The surface slope contours in the left column (Figs. 15(c) and (e)) have a relatively uniform spacing in the field of view except near the single clamp point at the bottom whereas the contours in the right column (Figs. 15(d) and (f)) show a distinct abnormality evident as pinched surface slope contours, attributed to the presence of disbond on the mid-plane of the disk in that region. Interestingly, the contours of $\frac{\partial w}{\partial x}$ in the well bonded disk show zero slope values coinciding with the vertical axis of symmetry of the disk when clamped at its lower edge. On the other hand, the corresponding contours of $\frac{\partial w}{\partial y}$ show a monotonically varying slope from the clamped lower edge to the free top edge of the disk, consistent with the imposed boundary condition. Interestingly, in case of the bi-layered plate with an embedded disbond, an unintended disbond during fabrication of the specimen is also seen vividly in the form of a second but smaller group of pinched slope contours at the upper top corner in Figs. 15(d) and (f). These results clearly demonstrate the ability of r-DGS for disbond detection applications.

\subsection{Damage Detection in Composite Plates}

Next, glass fiber reinforced composite plates (G10-FR4 from ePlastics, Inc.) of dimensions 76.2 $\mathrm{x} 76.2 \mathrm{~mm}^{2}$ and $3.1 \mathrm{~mm}$ thickness were used to demonstrate the ability of r-DGS to detect impact induced damage. As shown schematically in Fig. 16, the composite plate specimen was first clamped to a steel support with a clear aperture using C-clamps. The specimen was impacted using a long-bar impactor with a wedge shaped head. The impacting head contacted the composite plate over a rectangular region of $25.2 \times 8.2 \mathrm{~mm}^{2}$. The loading device consisted of an aluminum long-bar struck by an aluminum cylindrical striker propelled by compressed gas stored in a cylinder. The striker velocity was approximately 8 m/sec. A strain gage (CAE-13-125UN- 
120 from Vishay Micro-Measurements) bonded to the long-bar was used to measure the strain history in the bar during impact loading. The strain measurements were done using an oscilloscope and a signal amplifier/conditioner with a quarter Wheatstone bridge circuit. A measure strain signature from the long-bar is also shown in Fig. 16 for completeness.

After impacting the specimen, the composite plate was prepared for optical inspection. The face opposite to the impacted face of the composite plate was made reflective using film transfer technique, as detailed in Ref. 26. The steps involved were as follows: A PMMA plate was deposited with a thin layer of aluminum using a metal vapor deposition system. The surface of the composite to be made reflective was cleaned and a few uncured drops of two-part epoxy were placed on the specimen surface in the region of interest. The PMMA plate ( $\sim 9 \mathrm{~mm}$ thick) with the aluminum film was placed on the composite substrate (aluminized face in contact with the substrate) and excess epoxy was squeezed out using dead-weights. The excess epoxy along the edges of the PMMA plate were cleaned used a cotton swab. After the epoxy layer (typically a few microns thick) was cured, the PMMA plate was pried off the composite substrate. Since the bond strength between aluminum and epoxy exceeds that between aluminum and PMMA, the aluminum film was transferred to the composite specimen when the disk was pried off the substrate making it specularly reflective.

Thus prepared composite specimen was held vertically using the screw clamp in a setup shown in Fig. 14 and was subjected to thermal excitation by the incandescent lamp placed on the backside of the damaged sheet with the reflective side facing the camera. Similar to experiments with bi-layered samples, the speckle images were recorded first via the composite plate in the reference state prior to thermal excitation. Then the composite sheet was exposed to the incandescent lamp for a few minutes. As the epoxy matrix used in the composite sheet had a dark pigment, heat absorption was more efficient in these experiments when compared to the PMMA bi-layer disks. The image corresponding to the deformed state was hence recorded after a few minutes of cool-off period to ensure that the specimen surroundings were free of thermal currents. The speckle images ( 1 pixel covered $43 \mu \mathrm{m}^{2}$ on the target plane) were segmented into 25 x 25 pixel subsets with a 20 pixel overlap during image correlation. This yielded an array of $48 \times 66$ data points in the region of interest. Fig. 17 shows the two orthogonal surface slopes due to thermo-mechanical deformation of the damaged specimen. The manifestation of impact 
induced damage can be clearly seen from the surface slope contours. Even though no visible damage could be seen by the naked eye, the slope contours obtained clearly suggest the footprint of the rectangular impactor, seen from the opposite side (observer side) of the plate. The wedge impactor contact area being rectangular in shape, concentration of slope contours along the short-edges and at the four-corners of the rectangle is clearly evident. This again demonstrates the feasibility of the method for further quantitative analysis.

\section{Conclusions}

In this work the reflection-mode Digital Gradient Sensing (r-DGS) technique has been successfully extended to map stress-wave induced deformations in thin structures by coupling it with high-speed photography to extract two orthogonal surface slope components. The robustness of the method to perform measurements satisfactorily with different experimental parameters such as the coarseness of speckles used, target plane distances from the specimen, sub-image sizes and pixel overlap during image correlation have been demonstrated first. The out-of-plane displacements extracted from surface slopes based on numerical integration are in good agreement with a companion elasto-dynamic finite element analysis of the demonstration

problem namely a circular elastic plate subjected to central impact by a stiff spherical projectile. The feasibility of r-DGS to detect disbond and impact induced damage in layered solids is also demonstrated. An embedded disbond in a bi-layered acrylic disk and impact induced delamination in a fiber reinforced composite laminate have been successfully detected by mapping anomalies in the surface slope manifestations when the specimens were subjected to thermal excitation. Further work on the smallest measurable angular deflections and its accuracy using r-DGS are currently being pursued.

\section{Acknowledgments}

The partial support of this research by the National Science Foundation (grant \# CMMI1232821) and Department of Defense (grant \# W31P4Q-14-C-0049) are gratefully acknowledged.

\section{References}


1. F.K. Lichtenberg, "The moiré method: A new experimental method of the determination of moments in small slab models," Proc. SESA, 12, 83-98, 1955.

2. T.Y. Kao and F.P. Chiang, "Family of grating techniques of slope and curvature measurements for static and dynamic flexure of plates," Optical Engineering, 21(4), 721742 (1982).

3. R. Ritter, "Reflection moire methods for plate bending studies," Optical Engineering, 21(4), 63-671 (1982).

4. A. Assa, A.A. Betser and J. Politch, "Recording slope and curvature contours of flexed plates using gratings shearing interferometer," Applied Optics, 16(9), 2504-2513 (1977).

5. H.V. Tippur, S. Krishnaswamy, and A.J. Rosakis, "Optical Mapping of Crack Tip Deformations Using the Methods of Transmission and Reflection Coherent Gradient Sensing - a Study of Crack Tip K-Dominance,” International Journal of Fracture, 52(2), 91-117 (1991).

6. H. Lee, A.J. Rosakis and L.B. Freund, "Full-field optical measurement of curvatures in ultrathin- film-substrate systems in the range of geometrically nonlinear deformations," Journal of Applied Physics, 89, 6116-6129 (2001).

7. T.S. Park, S. Suresh, A.J. Rosakis and J. Ryu, "Measurement of full-field curvature and geometrical instability of thin film-substrate systems through CGS interferometry," Journal of the Mechanics of Physics and Solids, 51(11-12), 2191-2211 (2003).

8. H.V. Tippur, "Simultaneous and real-time measurement of slope and curvature fringes in thin structures using shearing interferometry," Optical engineering, 43(12), 3014-3020 (2004).

9. F.P. Chiang and R.M. Juang, "Laser speckle interferometry for plate bending problems," Applied Optics, 15, 2199-2204 (1976).

10. Y.Y. Hung and A.J. Durelli, "Simultaneous measurement of three displacement derivatives using a multiple image-shearing interferometric camera," Journal of Strain Analysis, 14, 81-88 (1978).

11. K. Patorski, "Generation of the derivative of out-of-plane displacements using conjugate shear and moire interferometry," Applied Optics, 25, 3146-3151 (1986).

12. B. Bhaduri, N.K. Mohan, and M.P. Kothiyal, Simultaneous measurement of out-of-plane displacement and slope using a multiaperture DSPI system and fast Fourier transform," Applied Optics, 46(23), 5680-5686, 2007. 
13. L. Huang, C. S. Ng, and A. K. Asundi, "Dynamic three-dimensional sensing for specular surface with monoscopic fringe reflectometry," Optics Express 19(13), 12809-12814 (2011).

14. Z. Liu, J. Guo, W. Shi, X. Huang, and H. Xie, "Transmission-speckle correlation for measuring dynamic deformation of liquid surface," Optics and Lasers in Engineering 65,110-116(2015).

15. T.C. Chu, W.F. Ranson, M.A. Sutton and W.H. Peters, "Application of digital image correlation techniques to experimental mechanics," Experimental Mechanics, 25(3), 232244 (1985).

16. D.J. Chen, F.P. Chiang, Y.S. Tan and H.S. Don, "Digital speckle-displacement measurement using a complex spectrum method," Applied Optics, 32(11), 1839-1849 (1993).

17. M.S. Kirugulige, H.V. Tippur and T.S. Denney, "Measurement of transient deformations using digital image correlation method and high-speed photography: application to dynamic fracture," Applied Optics, 46(22), 5083-5096 (2007).

18. B. Pan, K. Qian, H. Xie, and A. Asundi, "Two-dimensional digital image correlation for in-plane displacement and strain measurement: a review," Measurement science and technology 20 (6), 062001, 2009.

19. M.A. Sutton, U. Orteu, and H. Schreier, Image Correlation for Shape, Motion and Deformation Measurements, Springer (2009).

20. C. Periasamy and H.V. Tippur, "A full-field digital gradient sensing method for evaluating stress gradients in transparent solids,” Applied Optics, 51(12), 2088-2097 (2012).

21. C. Periasamy and H.V. Tippur, "Measurement of orthogonal stress gradients due to impact load on a transparent sheet using digital gradient sensing method,” Experimental Mechanics, 53(1), 97-111 (2013).

22. C. Periasamy, and H.V. Tippur, "A full-field reflection-mode digital gradient sensing method for measuring orthogonal slopes and curvatures of thin structures," Measurement Science and Technology, 24 (2), 025202, 2013.

23. A. Jain, "Extension of Reflection-Mode Digital Gradient Sensing Method to Study Stress-Wave Dominant Problems and Impact Induced Damage Detection,” M.S. Thesis, Auburn University, AL, 2015. 
24. A. Zerwera , M.A. Polaka, and J.C. Santamarina, 'Wave propagation in thin Plexiglas plates: implications for Rayleigh waves,' NDT\&E International 33, 33-41, 2000.

25. Ambu, R., F. Aymerich, F. Ginesu, and P. Priolo, "Assessment of NDT interferometric techniques for impact damage detection in composite laminates," Composites Science and Technology, 66(2),199-205, 2006.

26. H. V. Tippur, "Optical Techniques in Dynamic Fracture Mechanics," in Dynamic Fracture Mechanics, A. Shukla (Ed.), World Scientific, 2006. 


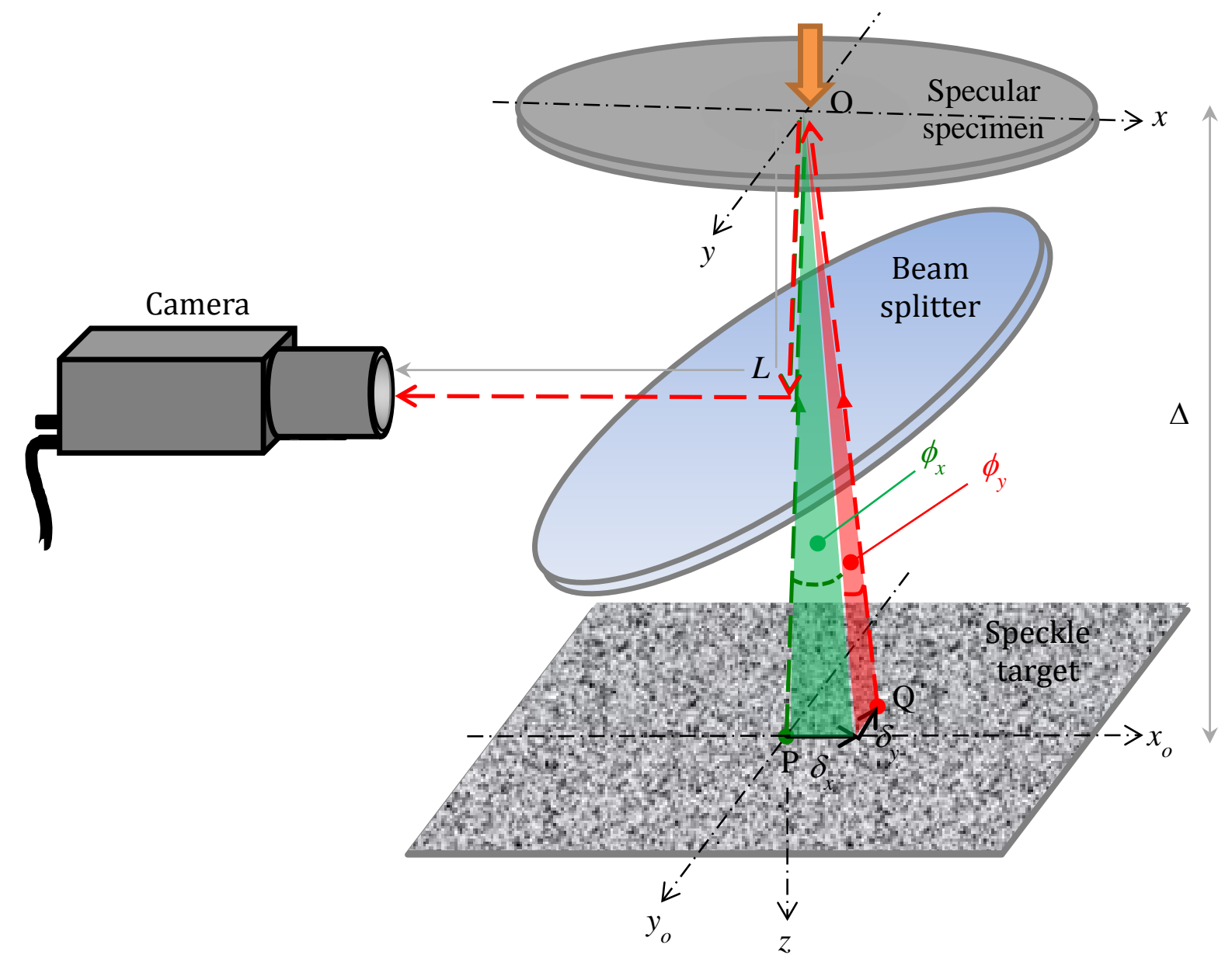

Figure 1: Schematic depicting the experimental setup and working principle of reflection-mode DGS (r-DGS) methodology. 

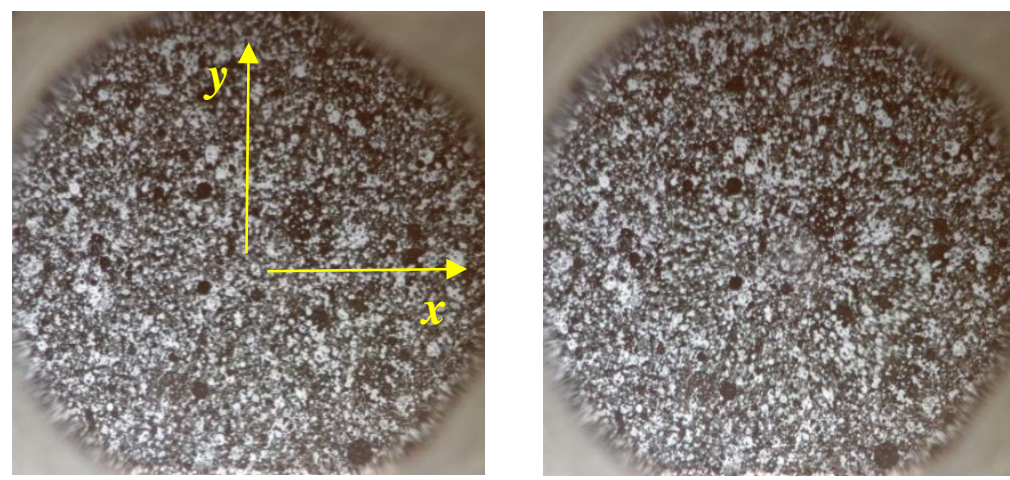

Figure 2: Recorded speckles from the target plane for r-DGS demonstration. (a) Reference image, (b) Deformed image with $30 \mu \mathrm{m}$ central deflection. ( $\Delta=65$ $\mathrm{mm}$, Si wafer diameter $50.8 \mathrm{~mm}$ )
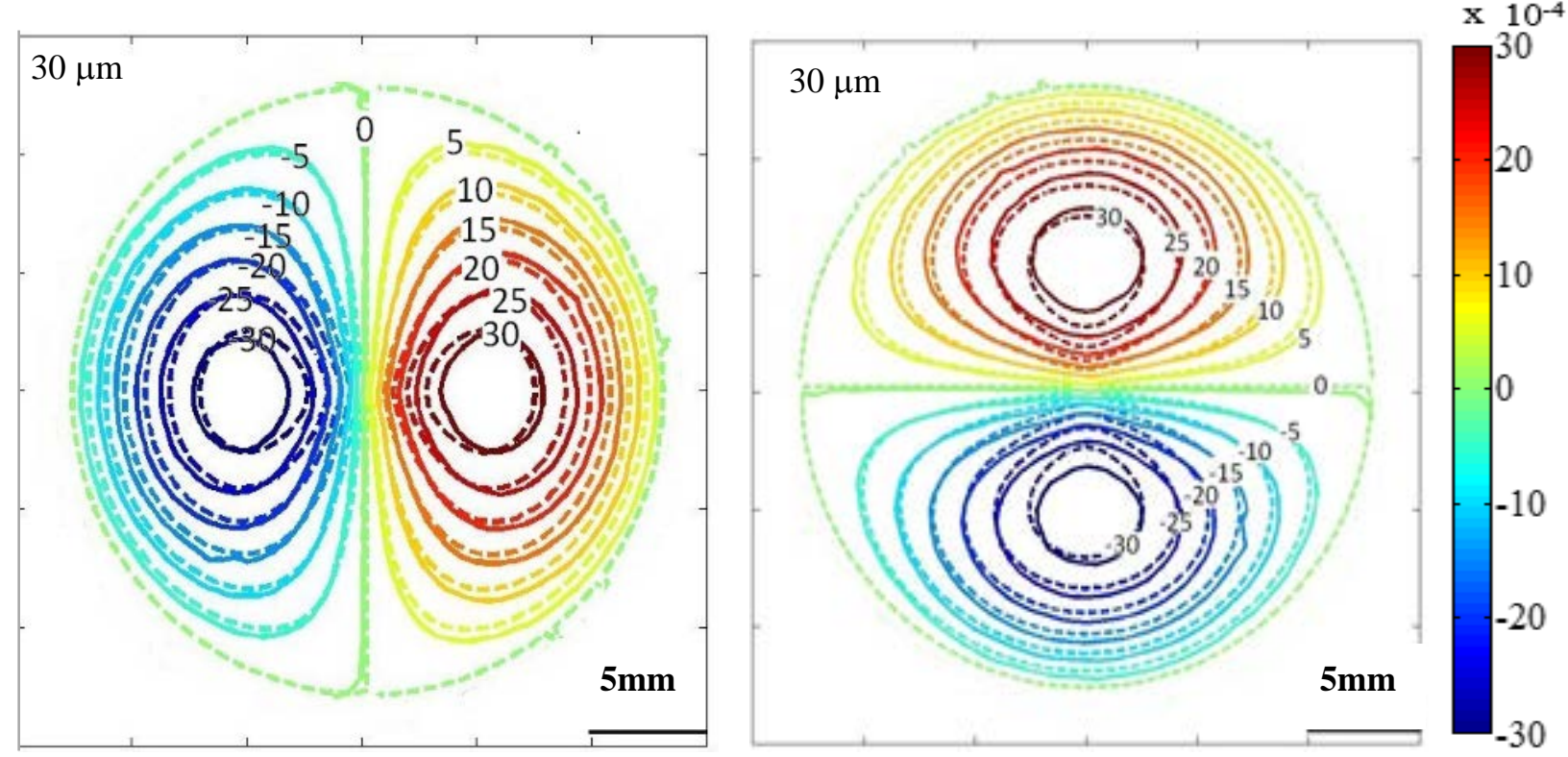

Figure 3: Experimental (solid lines) and analytical (broken lines) contours of surface slopes $\frac{\partial w}{\partial x}$ (left) and $\frac{\partial w}{\partial y}$ (right) for a clamped silicon wafer subjected to $30 \mu \mathrm{m}$ central displacement in the z-direction. Contour levels are in $5 \times 10^{-4}$ radian increments; $L=$ $1217 \mathrm{~mm}$ and $\Delta=65 \mathrm{~mm}$. 

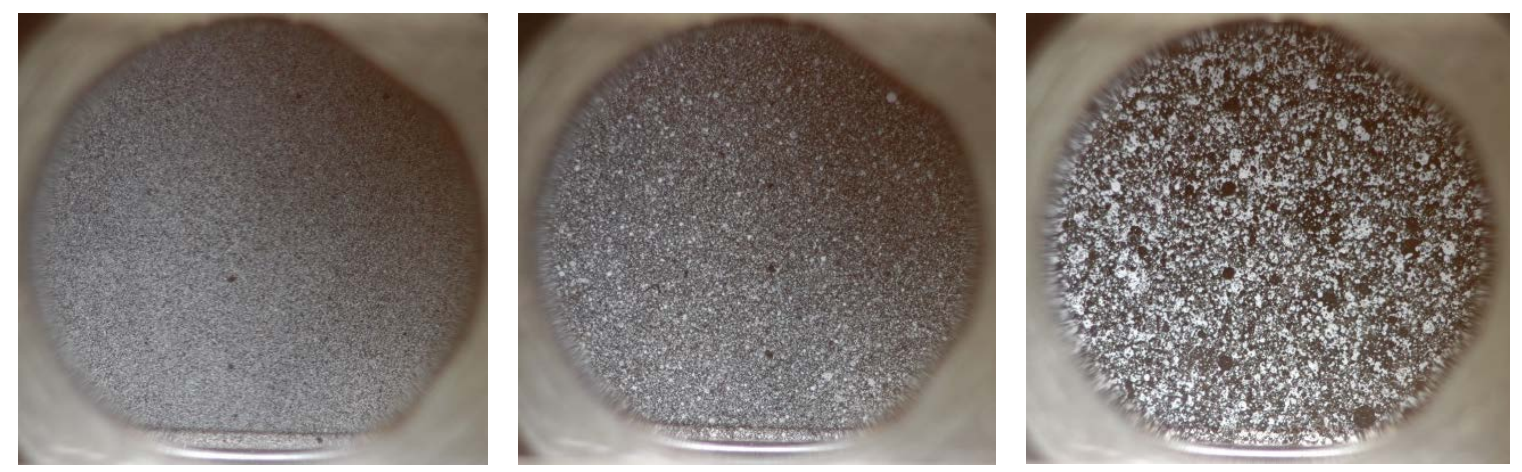

Figure 4: Three different speckle targets used in the baseline experiments: Fine (left), medium (middle), coarse (right) target speckles. (The straight edge of the silicon wafer is located at the top-right quadrant of each image; Diameter of the silicon wafer is $50.8 \mathrm{~mm}$ ). On average speckles covered 4-5 pixels, 11-12 pixels and 22-24 pixels for fine, medium and coarse cases. 
(a)
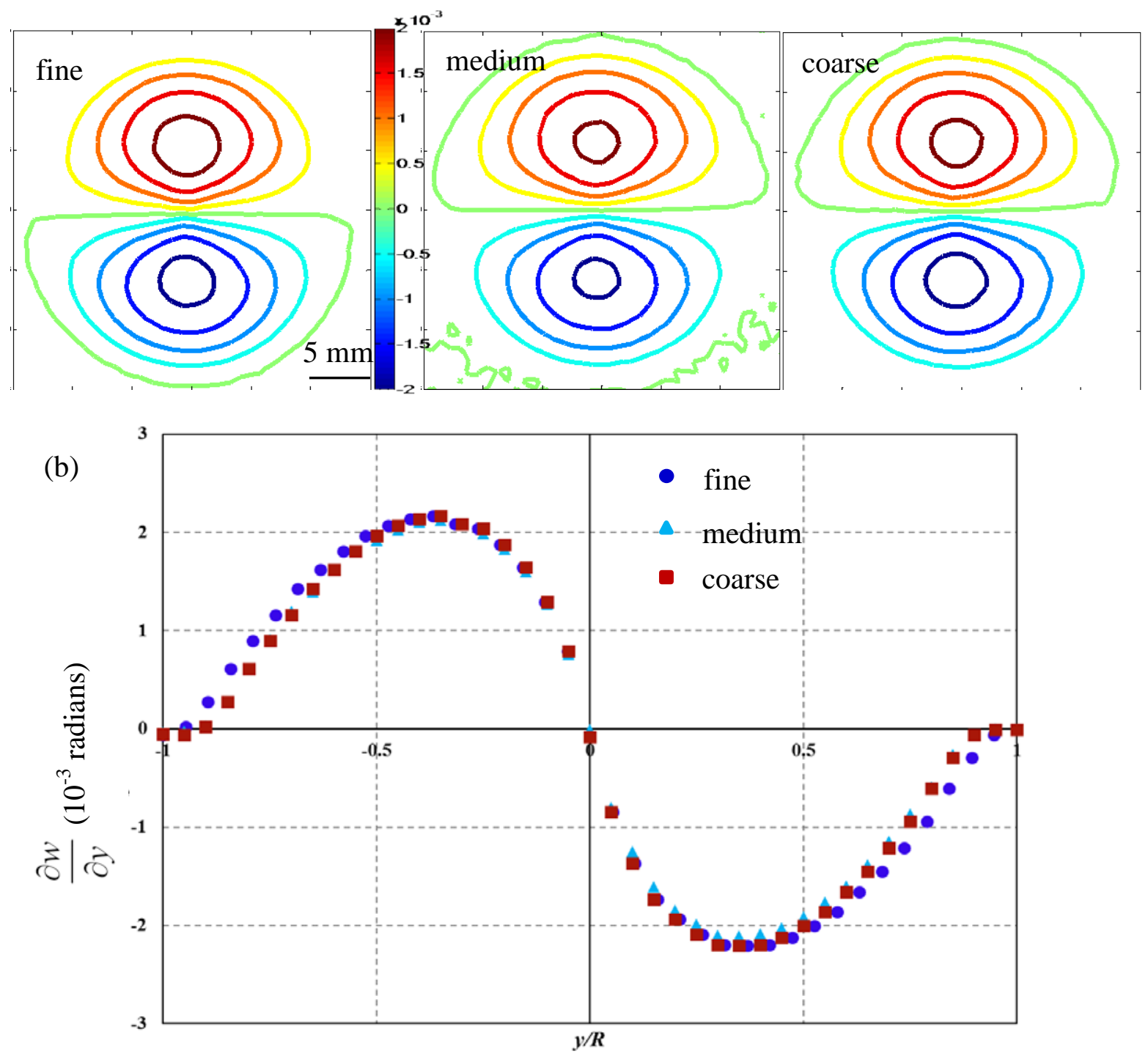

(c)

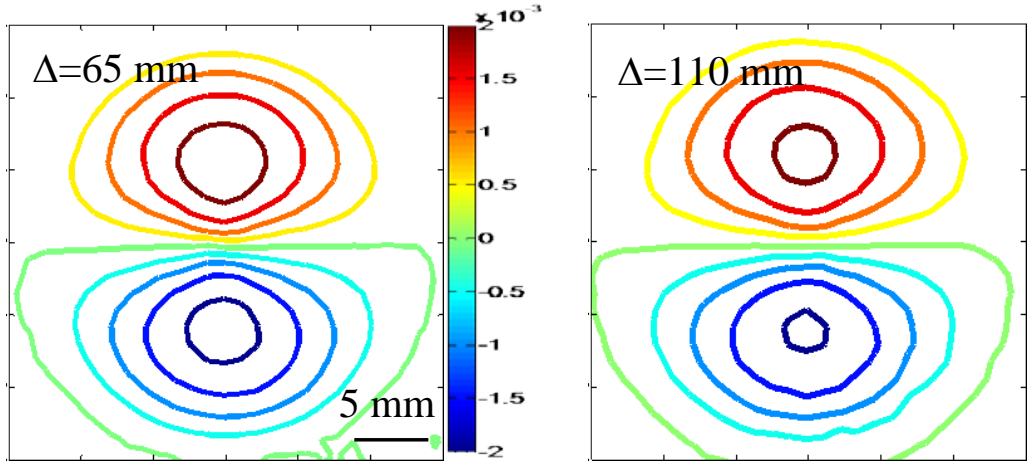

Figure 5: (a) Contours representing slopes $\frac{\partial w}{\partial y}$ for an applied displacement of $20 \mu \mathrm{m}$ and using fine, medium, coarse speckle patterns shown in Fig. $4(L=1217 \mathrm{~mm}$ and $\Delta=65 \mathrm{~mm})$. Contour levels are in $5 \times 10^{-4}$ radian increments. (b) Variation of $\frac{\partial w}{\partial y}$ along the vertical diameter of the silicon wafer for all the three speckle sizes. (c) Contours of $\frac{\partial w}{\partial y}$ for two different values of $\Delta, L=1217 \mathrm{~mm}$ and medium speckles. 

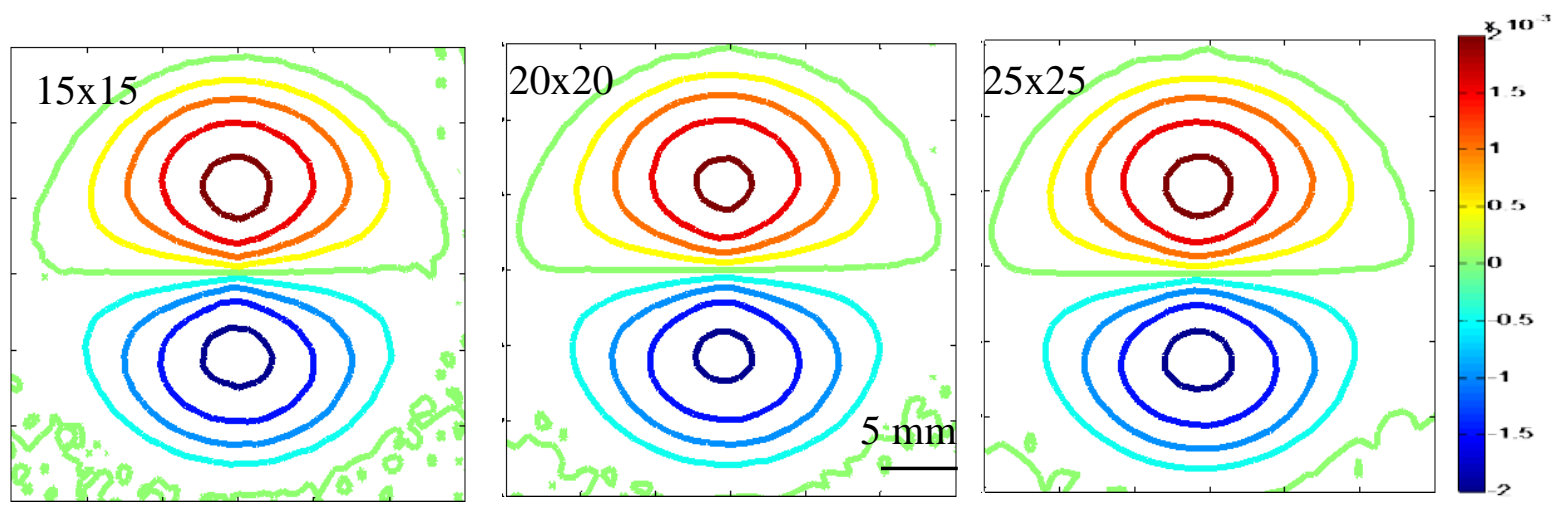

Figure 6: Surface slope contours of $\frac{\partial w}{\partial y}$ for an applied displacement of $20 \mu \mathrm{m}$ and medium size speckles on the target for different sub-image sizes without overlap. Contour levels are in $5 \times 10^{-4}$ radian increments. $L=1217 \mathrm{~mm}$ and $\Delta=$ $65 \mathrm{~mm}$ 

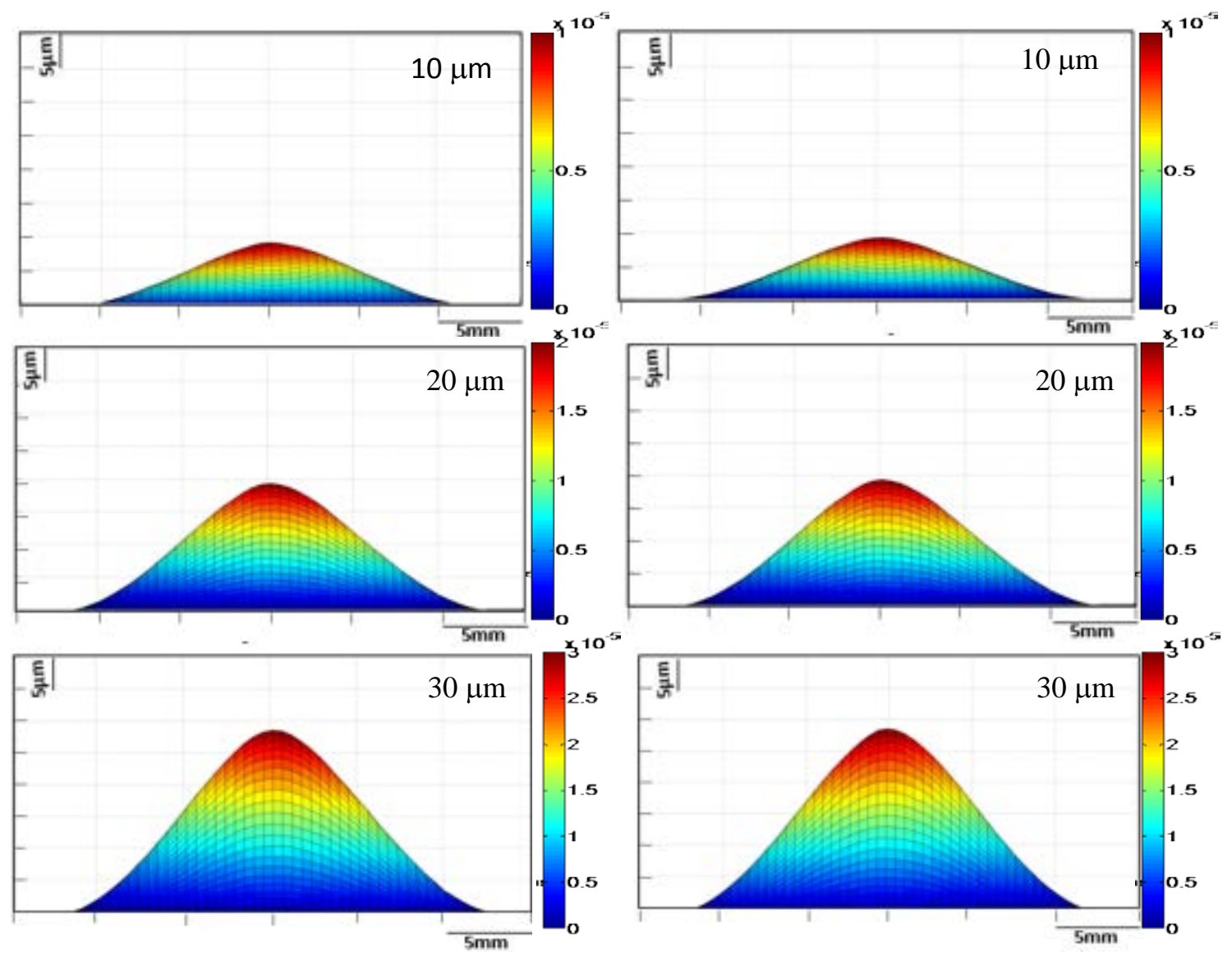

Figure 7: Plots of numerically integrated values of measured $\frac{\partial w}{\partial x}$ (left column) and $\frac{\partial w}{\partial y}$ (right column) for clamped silicon wafer subjected to central out-of-plane displacement of $10 \mu \mathrm{m}$, $20 \mu \mathrm{m}, 30 \mu \mathrm{m}$ (slope contours for this case shown in Fig. 3), respectively. (The color bar scale is in $10^{-5}$ meters.) 

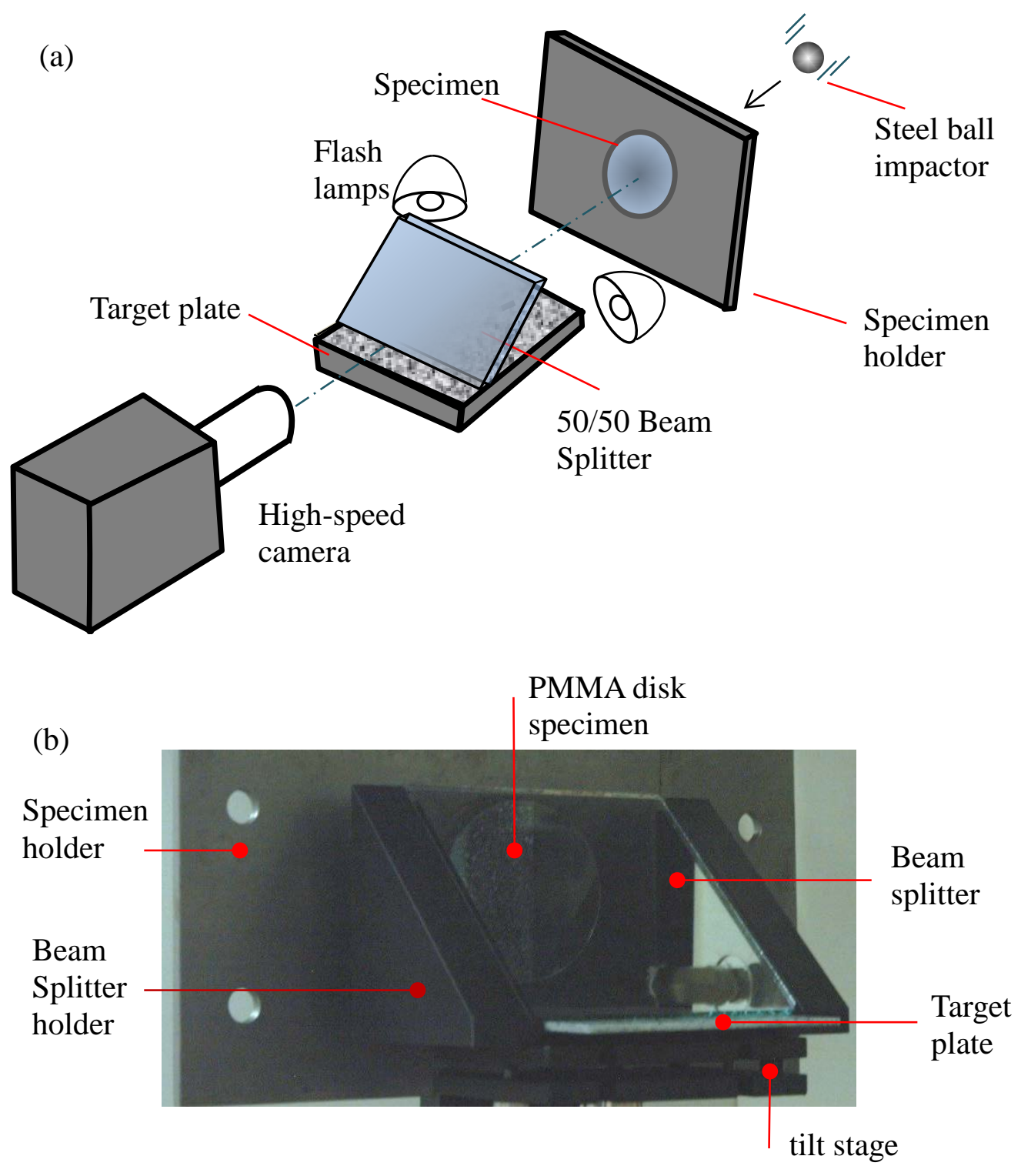

Figure 8: (a) Schematic of the experimental setup used for measuring surface gradients due to transient stress wave propagation in the disk due to impact, (b) Photograph of the specularly reflective PMMA disk behind the beam splitter/target plate. 

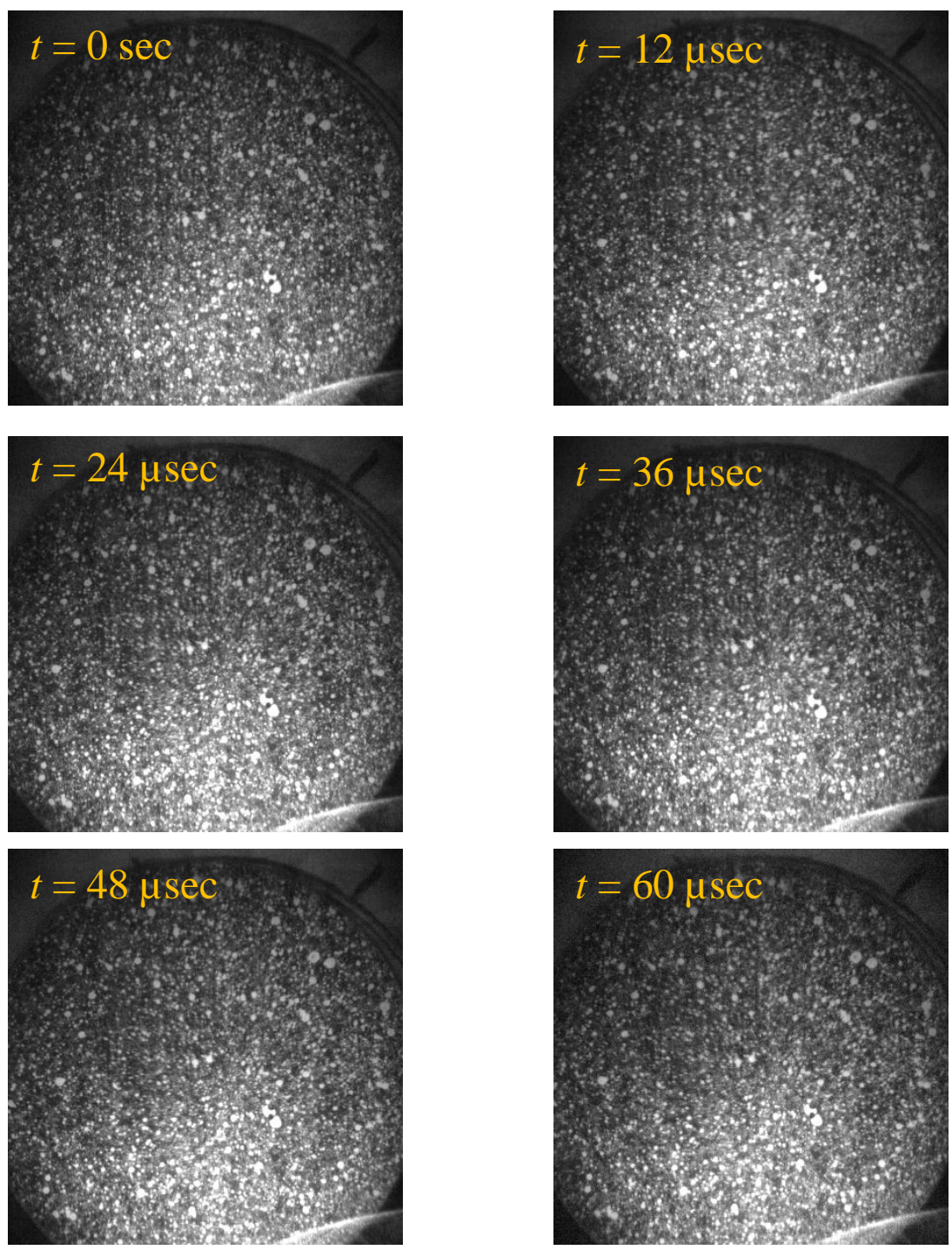

Figure 9: Recorded speckle images from Cordin-550 high-speed camera at different time instants after impact by the spherical ball at its center. The nominal diameter of the disk is $75 \mathrm{~mm}$. 

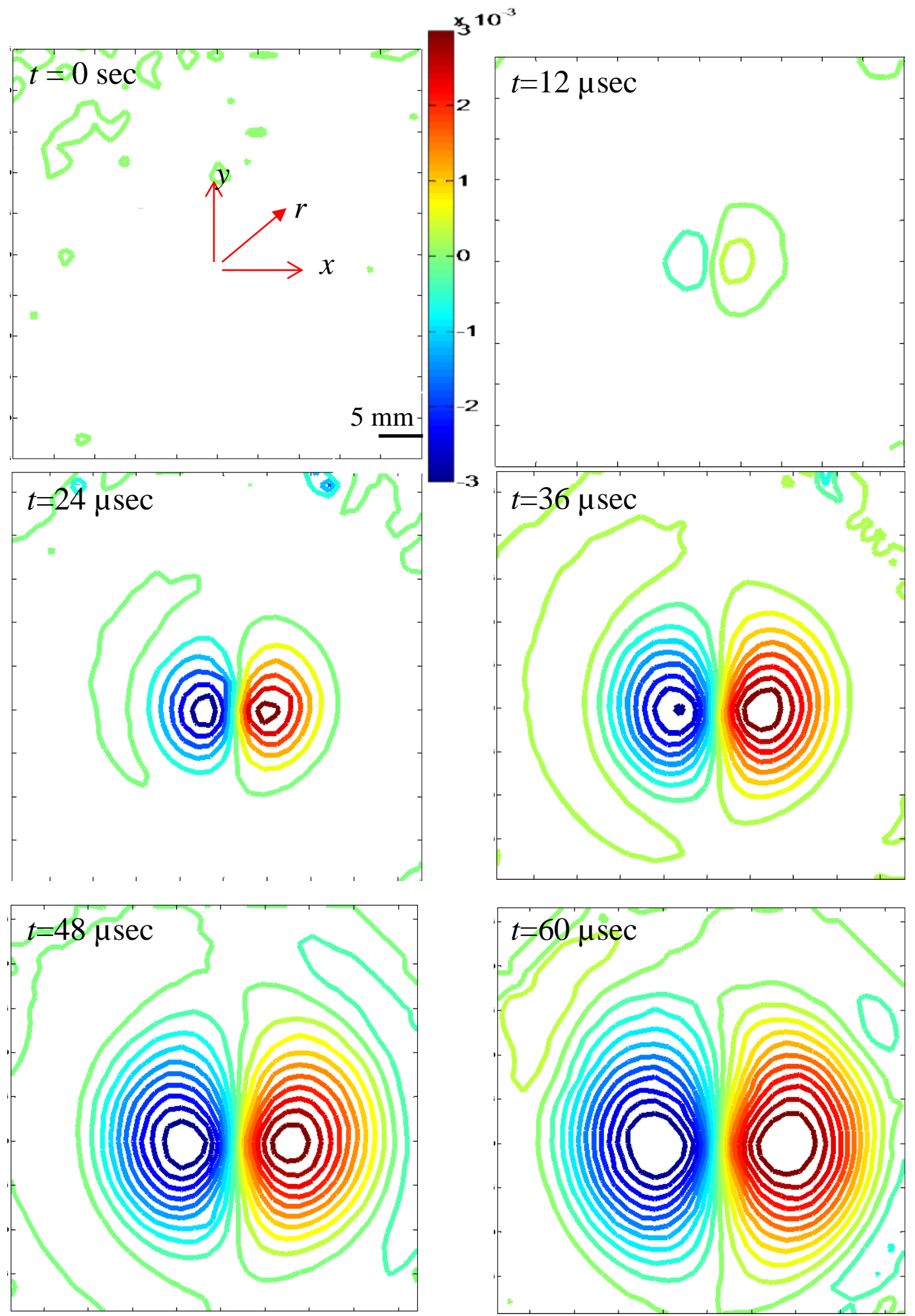

Figure 10: (a) Evolution of $\phi_{x}$ contours due to impact of a reflective PMMA disk by a steel ball. The contour levels are in $3 \times 10^{-4}$ radian increments. 


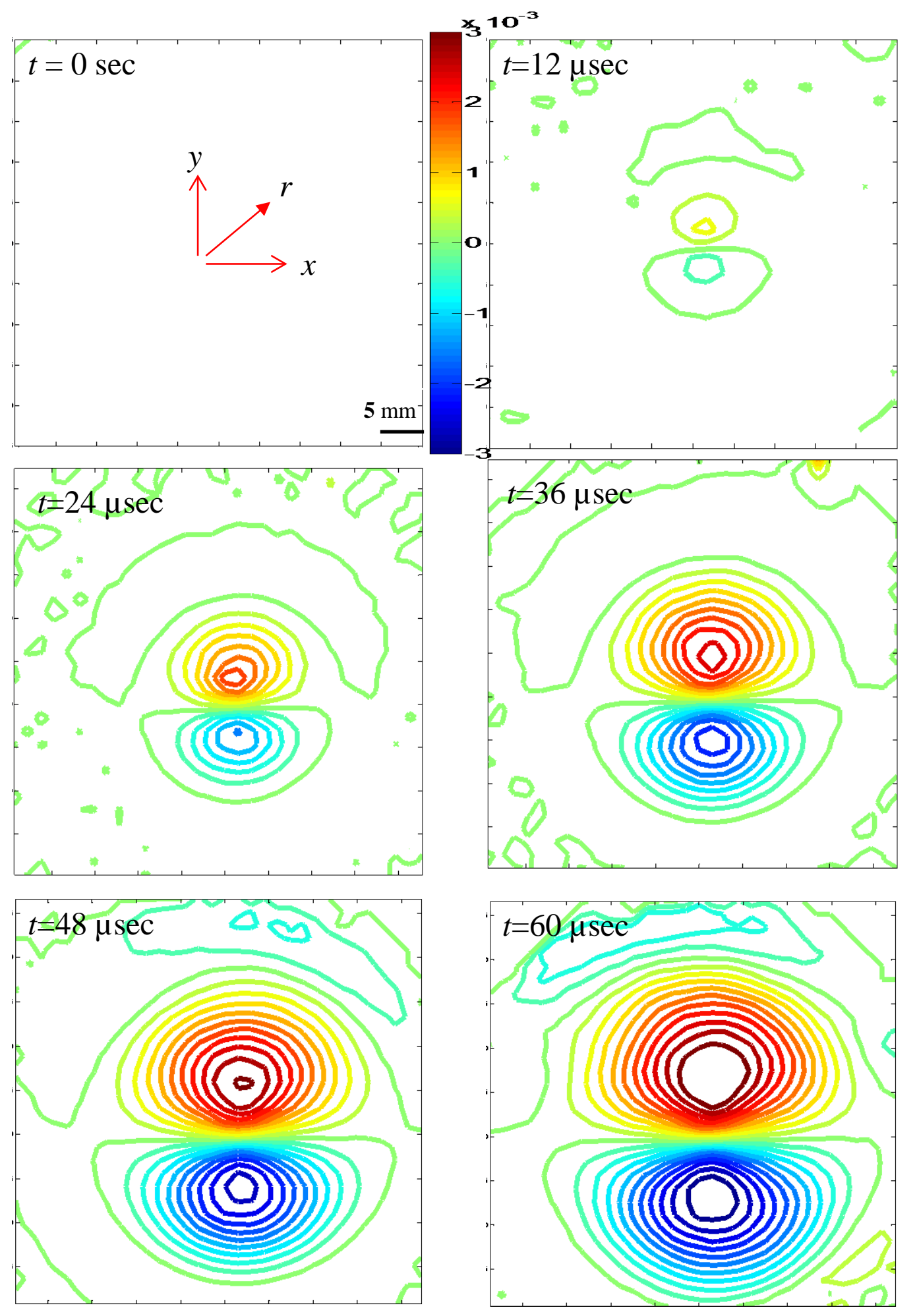

Figure 10: (b) Evolution of $\phi_{v}$ contours due to impact of a reflective PMMA disk by a steel ball. The contour levels are in $3 \times 10^{-4}$ radian increments. 

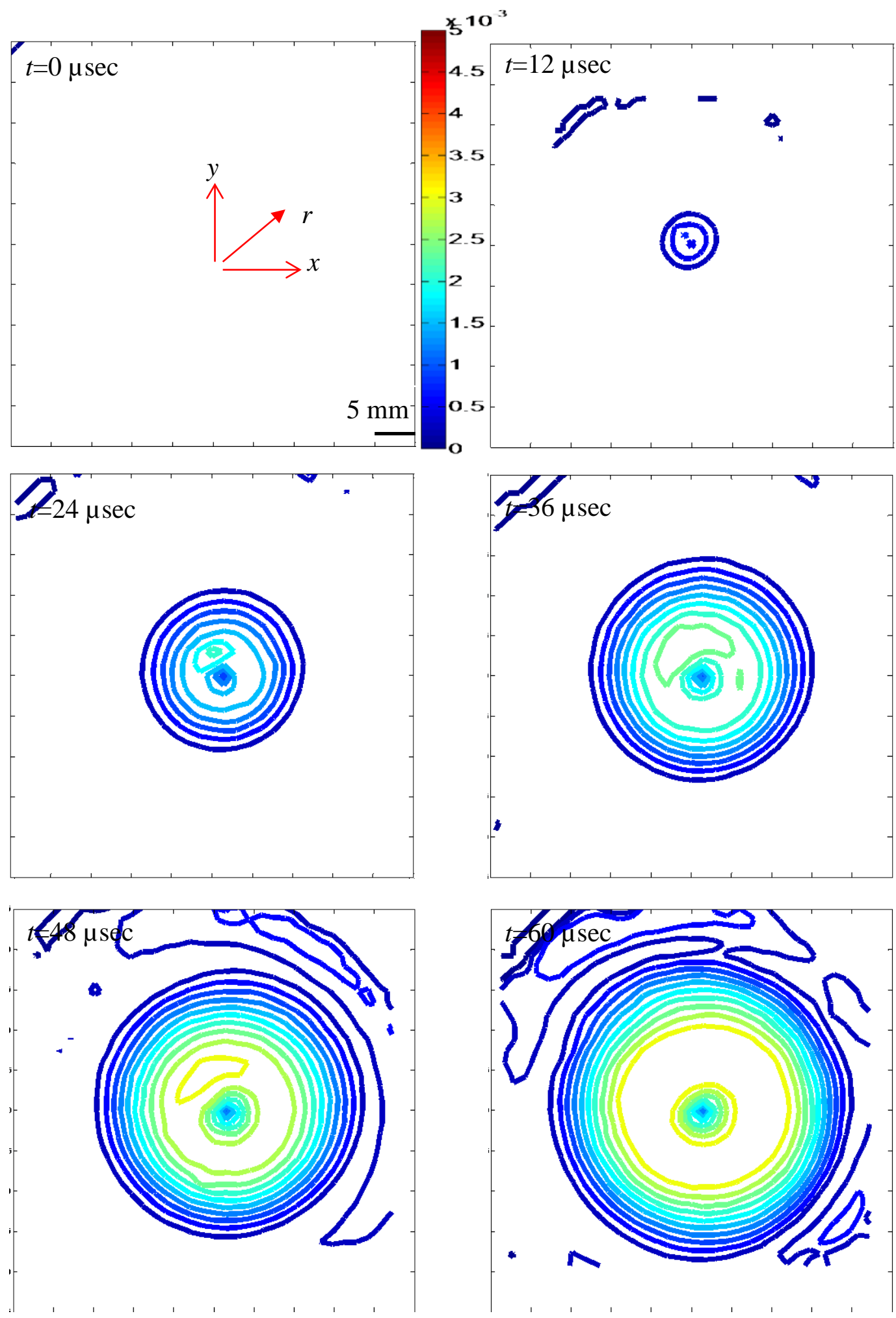

Figure 11: Evolution of $\phi_{r}\left(=\sqrt{\phi_{x}^{2}+\phi_{y}^{2}}\right)$ contours due to impact of a reflective PMMA disk by a steel ball showing the axisymmetric nature of deformations. The contour levels are in $3 \times 10^{-4}$ radian increments. 

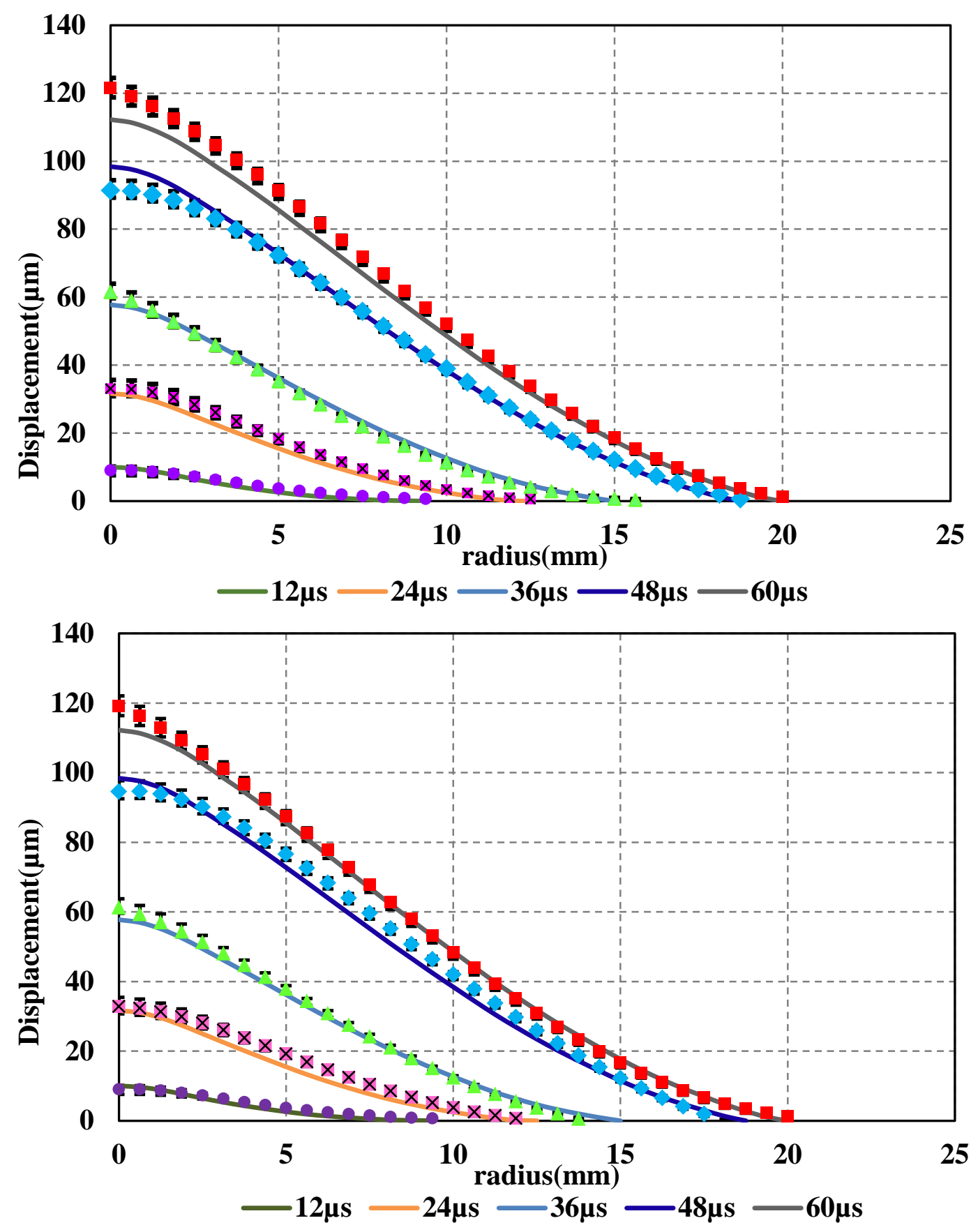

Figure12: Variation of out-of-plane displacements $(w)$ along $(x, y=0)$ (top) and $(x=0, y)$ (bottom) at different time instants after impact - solid symbols. Solid lines represent values from elasto-dynamic 3D finite element simulations. 


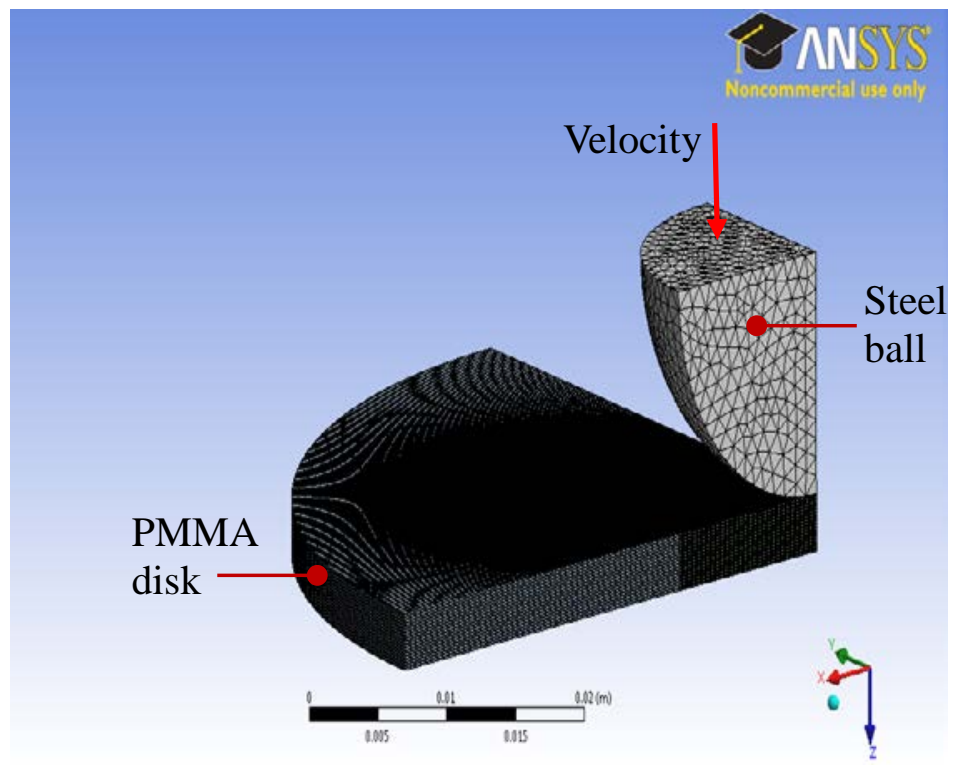

Figure 13: 3D Finite element model (quarter model) of ball impact on the PMMA plate. 


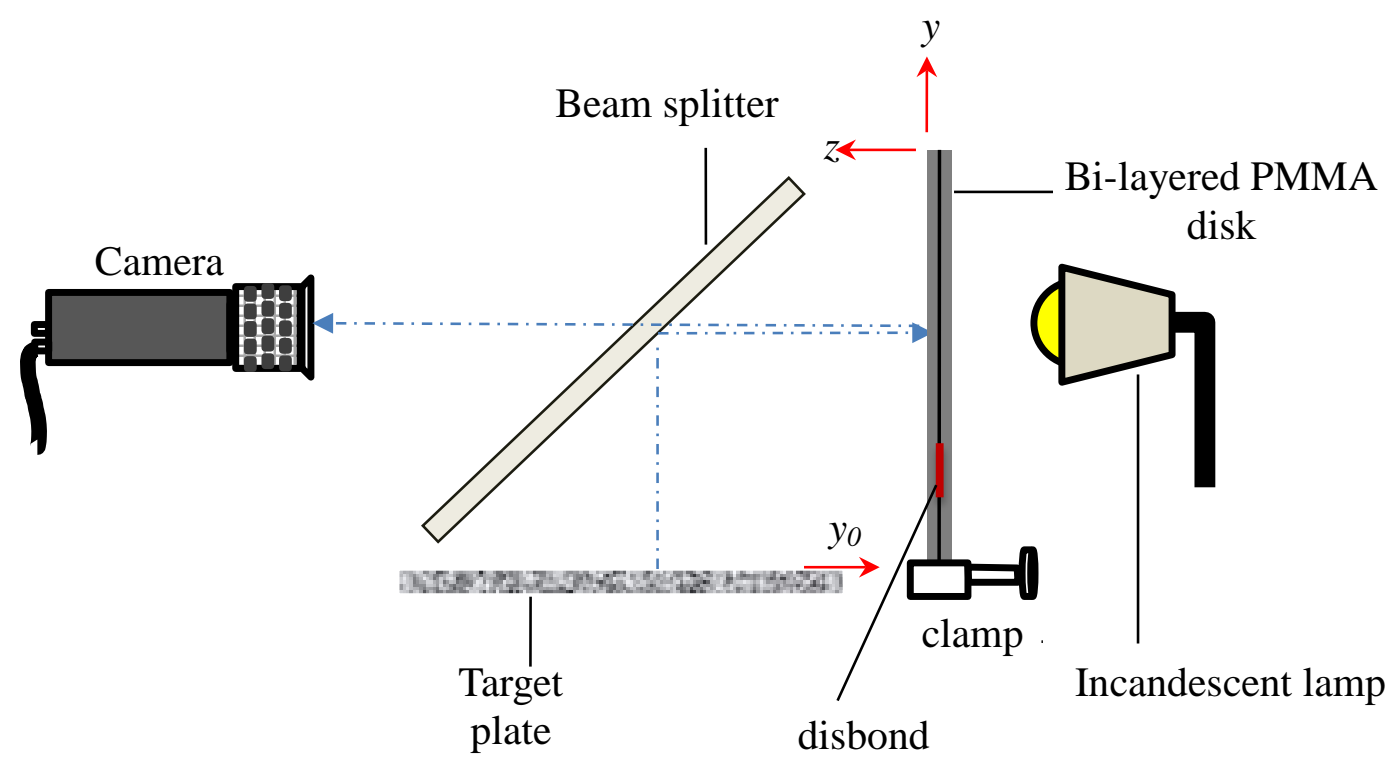

Figure 14: 2D schematic of the experimental setup used for debond detection using r-DGS. 


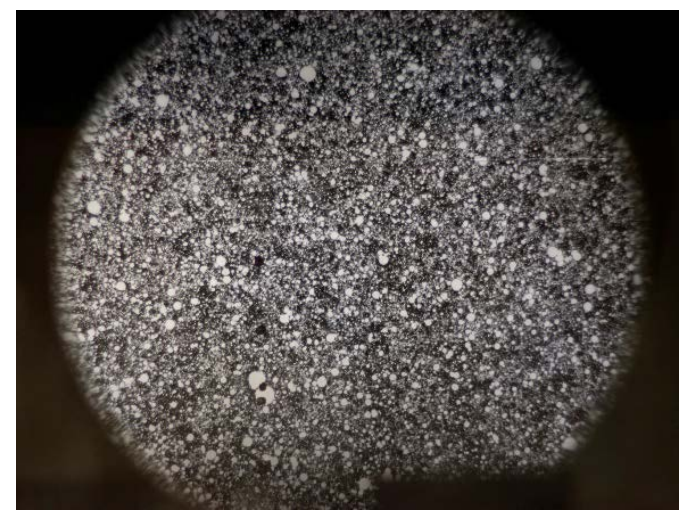

(a)

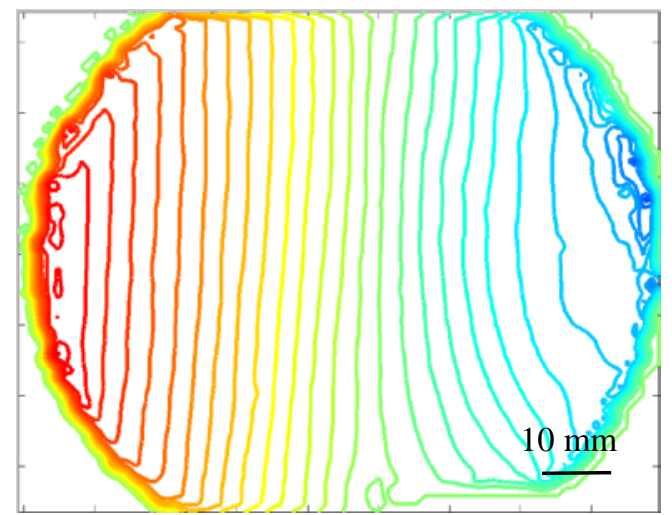

(c)

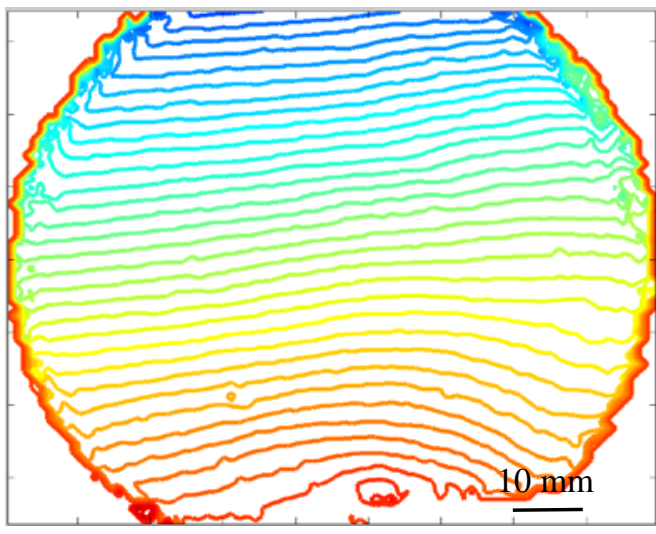

(e)

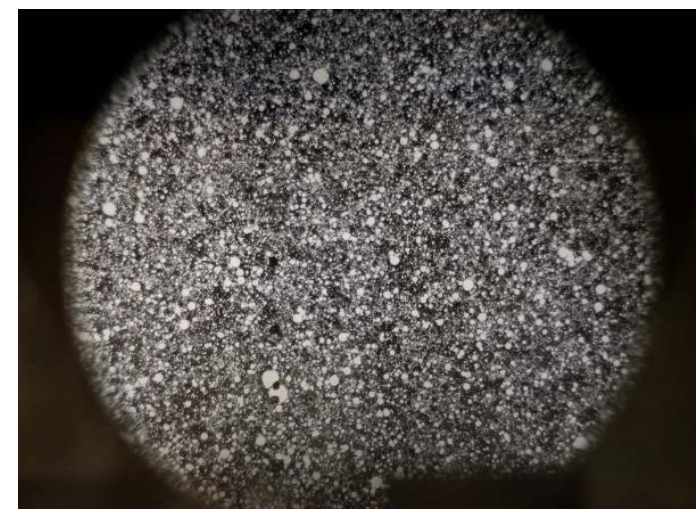

(b)
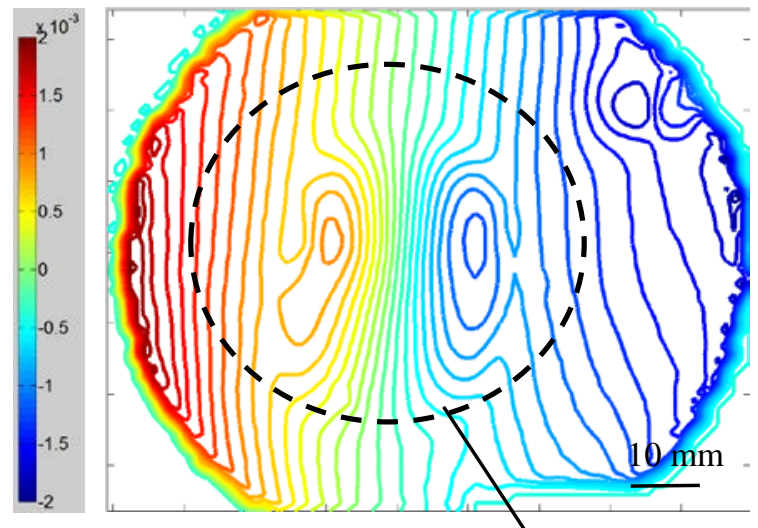

(d)
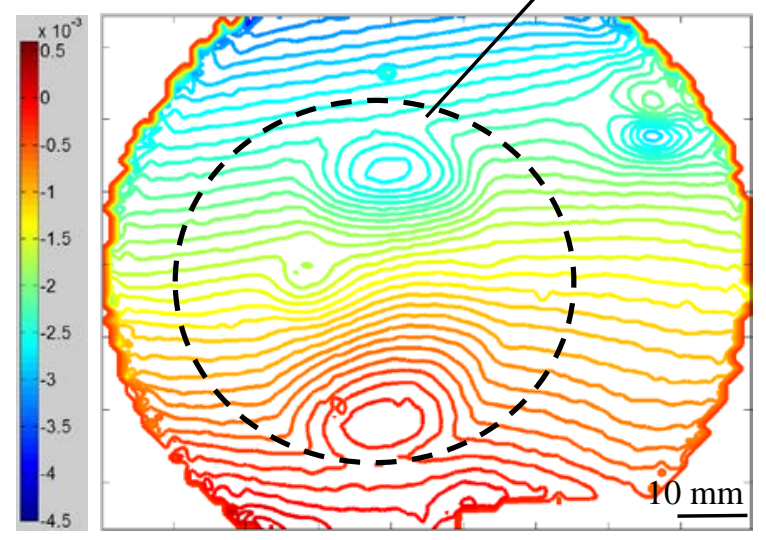

(f)

Figure 15: Reference (a) and deformed (b) images of target speckles recorded via a bilayered PMMA plate after 15 minutes of thermal excitation. Surface slope contours $\left(5 \times 10^{-4}\right.$ rad increment) on the front surface representing $\frac{\partial w}{\partial x}$ and $\frac{\partial w}{\partial y}$ for plates without (c, e) and with (d, f) disbond. Manifestation of the disbond in the form of 'pinched' contours is highlighted by the dotted circle in ( $d, f$ ). (A smaller unintended disbond at the top right corner of the disk in (d, f) is also evident.) 

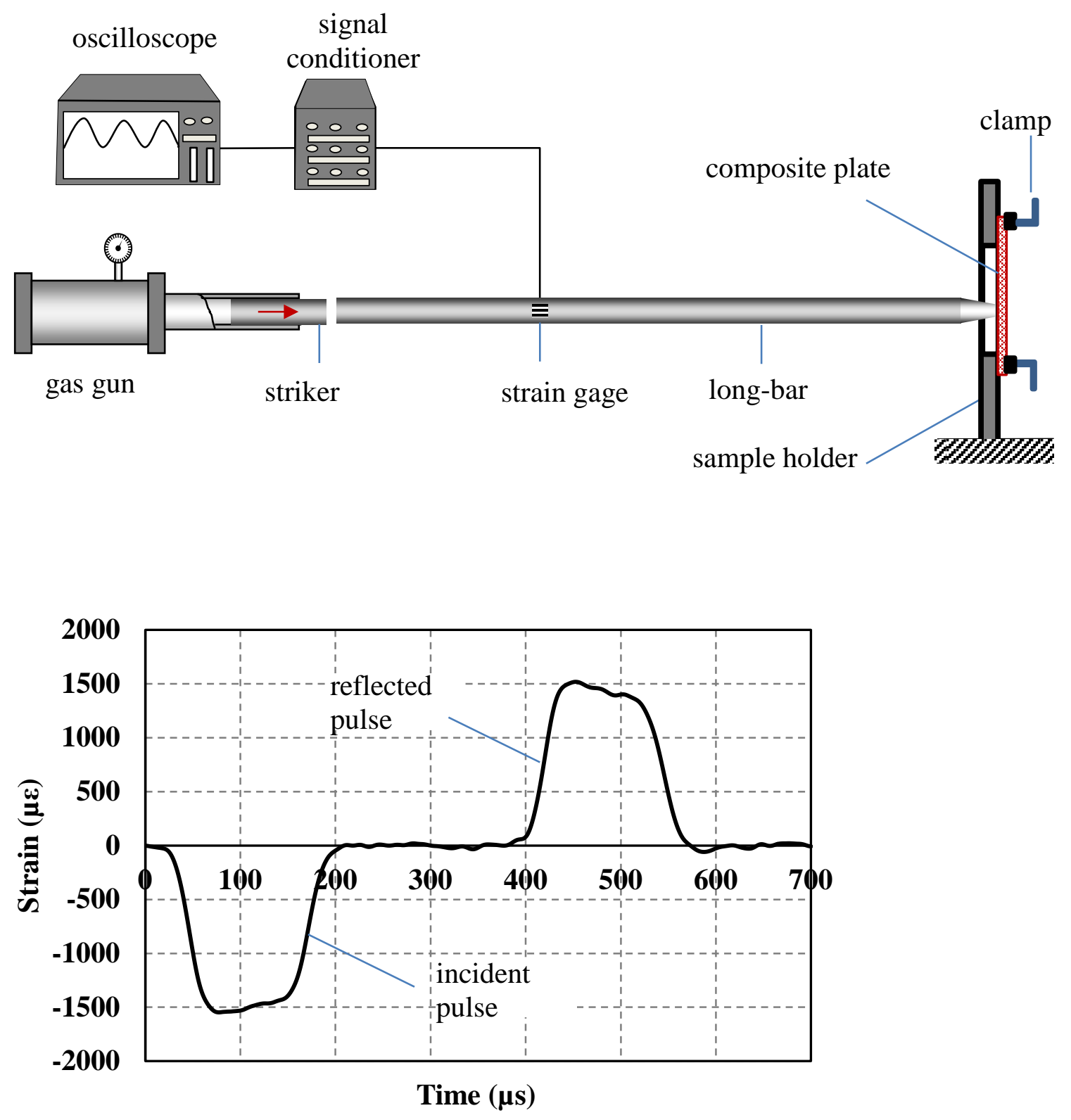

Figure 16: Schematic of impact loading setup (top) used to produce controlled damage on composite plate. The resulting strain signature (bottom) measured on the long-bar. 

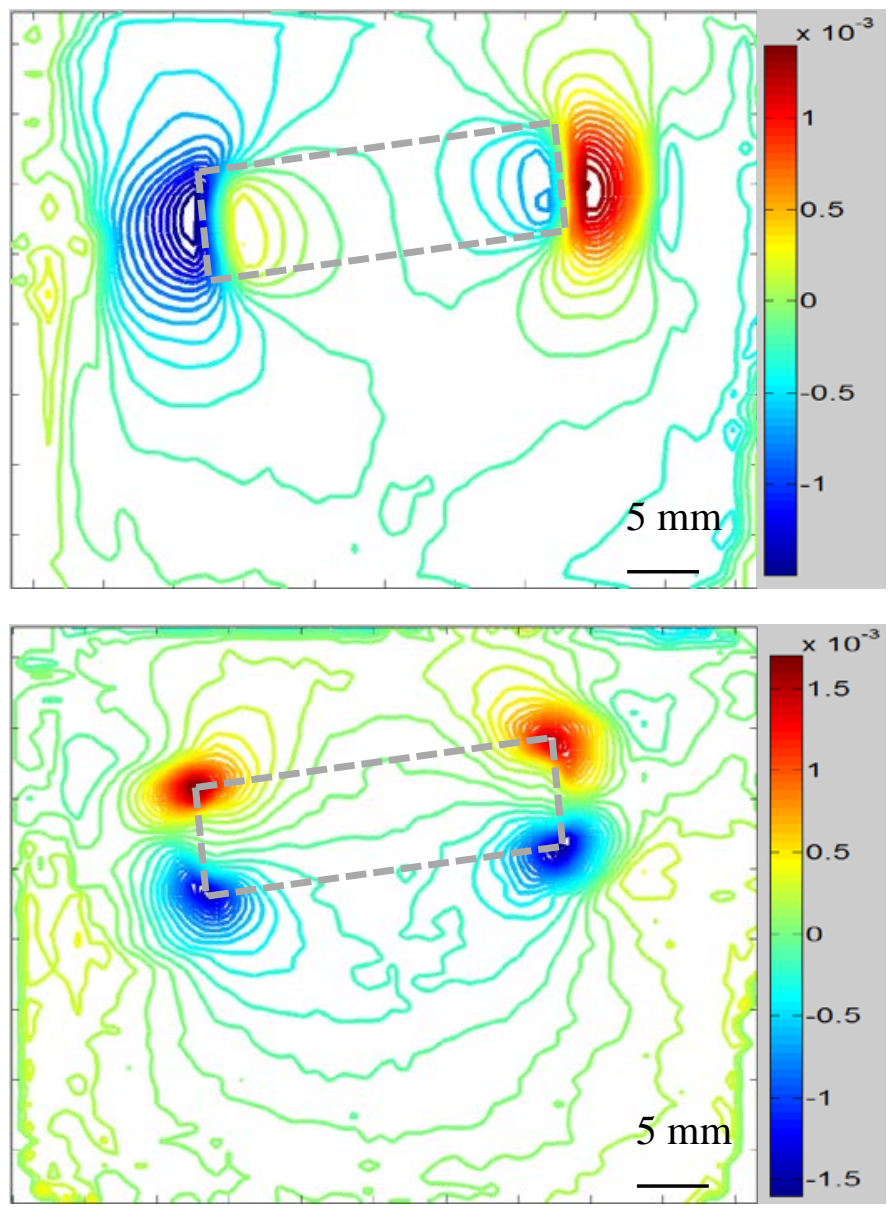

Figure 17: Surface slope contours $\frac{\partial w}{\partial x}$ (top) and $\frac{\partial w}{\partial y}$ (bottom) following 15 minutes of thermal excitation and 5 minutes of cool-down. Delamination zone is highlighted in the figure by the (gray) dotted rectangle corresponding to the footprint of the wedge impactor on back surface of the plate. 